\title{
Las medidas de tendencia central en libros de texto de Educación Primaria en México
}

\author{
Danilo Díaz-Levicoy \\ dddiaz01@hotmail.com \\ https://orcid.org/0000-0001-8371-7899 \\ Universidad Católica del Maule (UCM) \\ Talca, Chile. \\ Lizzet Morales-Garcia \\ lmgarcia@uagro.mx \\ http://orcid.org/0000-0002-2295-2278 \\ Universidad Autónoma de Guerrero (UAGro) \\ Guerrero, México. \\ Francisco Rodríguez-Alveal \\ frodriguez@ubiobio.cl \\ http://orcid.org/0000-0002-9141-4502 \\ Universidad de Bío-Bío (UBB) \\ Chillán, Chile.
}

Recibido: 15/04/2020 Aceptado: 15/05/2020

\section{Resumen}

En este trabajo se describen los principales resultados de analizar las actividades en que intervienen las medidas de tendencia central en libros de texto de Educación Primaria en México. Para ello, se realiza un análisis de contenido en los libros de $4^{\circ}, 5^{\circ}$ y $6^{\circ}$ de primaria que entrega la Secretaría de Educación Pública y los de una editorial privada, seis textos en total. Los resultados muestran el predominio de la media aritmética y la moda, la tarea de calcular, el uso de las representaciones de listado de datos y tabla de datos y el contexto personal; en el caso de la forma de trabajo, ésta depende de la editorial. Se observa la necesidad de aumentar la cantidad de las actividades sugeridas en los libros de texto.

Palabras clave: Libro de texto. Media aritmética. Moda. Mediana. Actividades.

\section{As medidas de tendência central nos livros didáticos da Educação Primária no México}

\section{Resumo}

Este trabalho descreve os principais resultados da análise das atividades nas quais medidas de tendência central intervêm nos livros didáticos da educação primária no México. Para isso, realizou-se uma análise de conteúdo nos livros da $4^{\mathrm{a}}, 5^{\mathrm{a}}$ e $6^{\mathrm{a}}$ séries do ensino fundamental distribuídos pela Secretaria da Educação Pública, e nos de uma editora privada, num total de seis textos. Os resultados mostram a predominância da média aritmética e da moda, a tarefa de calcular, o uso das representações de lista de dados e tabela de dados e o contexto pessoal; no caso da forma de trabalho, depende da editora. Observa-se a necessidade de aumentar o número de atividades sugeridas nos livros didáticos.

Palavras chave: Livro didático. Média aritmética. Moda. Mediana. Atividades. 


\title{
Measures of central tendency in textbooks of Primary Education from Mexico
}

\begin{abstract}
This article describes the main results of analyzing the activities in which the central tendency measures in Mexican textbooks of Primary Education are involved. To do this, a content analysis is carried out in books of 4th, 5th and 6th grade of primary education provided by the Public Education Secretariat and also those provided by a private publishing house, making six texts in total. The results show the predominance of the arithmetic mean and the mode, the calculating task, the use of data list representations and data table and the personal context; regarding the way of work, it depends on the publisher. The need to increase the amount of suggested activities in textbooks is observed.
\end{abstract}

Keywords: Textbook. Arithmetic mean. Mode. Median. Activities.

\section{Introducción}

En la actualidad, el uso de la estadística y sus elementos se han masificado debido a su potencial para comprender fenómenos en diversas áreas de conocimiento y en la vida cotidiana. En este sentido, los gráficos, las tablas y las medidas de resumen estadísticos permiten mostrar en forma sintetizada gran cantidad de información, la que debe ser entendida por los ciudadanos que están expuestos a multiplicidad de estos elementos en los medios de comunicación (periódicos, televisión, revistas, radio, etc.). En este sentido, el ciudadano necesita herramientas para leer e interpretar la información, y, partir de esto, poder opinar y tomar decisiones. Por otro lado, las directrices curriculares en el área de matemática en diferentes países incluyen contenidos de estadística en los diferentes niveles educativos (Batanero, 2001; Font, 2008), motivados por su utilidad e importancia.

Con respecto a las medidas de tendencia central (MTC), diversos autores señalan su importancia en la construcción y comprensión de otros conceptos estadísticos (e.g., Batanero, 2001; Cobo, 2003; Escobedo y Mayén, 2018; Mayén, 2009).

Las MTC, así como las tablas y gráficos estadísticos, son consideradas parte de la cultura estadística (Arteaga, Batanero, Cañadas y Contreras, 2011; Contreras y Molina-Portillo, 2019). Esta expresión hace referencia a un derecho ciudadano, que implica:

(...) leer e interpretar tablas, gráficos y medidas de resumen que aparecen en los medios; interpretar, evaluar críticamente y comunicar información estadística; comprender y utilizar el lenguaje y las herramientas básicas de la estadística; apreciar el valor de la estadística en la vida cotidiana, la vida cívica y la vida profesional en calidad de consumidor de datos, de modo de actuar como un ciudadano informado y crítico en la sociedad basada en la información (Del Pino y Estrella, 2012, p. 55). 
Otro elemento central en nuestro trabajo es el libro de texto, recurso pedagógico central para los procesos de enseñanza y aprendizaje de la matemática, producto de una transposición didáctica (Chevallard, 1991), usado ampliamente por profesores y estudiantes (Díaz-Levicoy, Giacomone y Arteaga, 2017; Díaz-Levicoy, Vásquez y Molina-Portillo, 2018), configurándose “como una invariante de la escuela, como un material estable, de hecho, el material pedagógico de más larga duración en la historia de la escuela, aunque sujeto a modificaciones y transformaciones" (Braga y Belver, 2016, p. 200). Además, guarda relación directa con las directrices curriculares (Herbel, 2007), porque es visto por los profesores como su representación en el aula (Salcedo, Molina-Portillo, Ramírez y Contreras, 2018) y contribuye al éxito o fracaso de su implementación (Cantoral, Montiel y Reyes-Gasperini, 2015).

De acuerdo con estas consideraciones, nos planteamos como objetivo: analizar las actividades sobre MTC presentes en libros de texto de matemática de Educación Primaria en México. Proporcionando información novedosa y de interés para quienes investigan en Didáctica de la Estadística en México y Latinoamérica.

Este trabajo se ha estructurado en los siguientes apartados: en la Sección 2 se sinteriza lo estipulado en las directrices curriculares sobre las MTC, en la Sección 3 se presentan resultados de investigaciones previas, en la Sección 4 describimos aspectos sobre la metodología del estudio, en la Sección 5 presentamos los resultados y, finalizamos, en la Sección 6 con las conclusiones más destacadas del trabajo de investigación.

\section{Las medidas de tendencia central en las directrices curriculares}

La estadística y la probabilidad son temas que se trabajan desde el inicio de la Educación Primaria en diferentes países (e.g., MECD, 2014; MINEDU, 2017; MINEDUC, 2012).

En México, siguiendo esta tendencia, en el Plan de Estudio (SEP, 2011a) y Programas de Estudio de Educación Primaria (SEP, 2011b; SEP, 2011c; SEP, 2011d), el estudio de las MTC se aborda de $4^{\circ}$ a $6^{\circ}$ grado en el eje Manejo de la información; el cual se tiene como objetivo "identificar las medidas de tendencia central en un conjunto de datos" (SEP, 2011b, p. 63). En el Cuadro 1 se presentan los contenidos relacionados con este tema por grado. 
Cuadro 1. Contenidos sobre las MTC para la Educación Primaria en México

\begin{tabular}{|c|l|}
\hline Grado & \multicolumn{1}{c|}{ Contenido } \\
\hline $4^{\circ}$ & $\begin{array}{l}\text { Identificación y análisis de la utilidad del dato más frecuente de un conjunto de datos } \\
\text { (moda) (SEP, 2011b, p. 78). }\end{array}$ \\
\hline $5^{\circ}$ & $\begin{array}{l}\text { Cálculo de la media (promedio) y análisis de su pertinencia respecto a la moda como } \\
\text { dato representativo en situaciones diversas (SEP, 2011c, p. 80). }\end{array}$ \\
\hline $6^{\circ}$ & $\begin{array}{l}\text { Uso de la media (promedio), la mediana, y la moda en la resolución de problemas } \\
\text { (SEP, 2011d, p. 77). }\end{array}$ \\
\hline
\end{tabular}

Fuente: elaborado por los autores

Actualmente, se están realizando cambios en los programas de estudio en México; mismos que se han plasmado paulatinamente en el Nuevo Modelo Educativo 2018 (SEP, 2017). En él, la enseñanza de las MTC se proponen de $4^{\circ}$ a $6^{\circ}$ curso de Educación Primaria, en el eje temático Análisis de datos, cuyo objetivo es "propiciar que los estudiantes adquieran conocimientos y desarrollen habilidades propias de un pensamiento estadístico y probabilístico" (SEP, 2017, p. 306). Este eje se organiza en cuatro ideas fundamentales, donde la tercera se relaciona directamente con las MTC: "el uso de medidas de tendencia central y de dispersión para reducir la complejidad de los conjuntos de datos y aumentar las posibilidades de operar con ellos" (Op. cit., p. 306). En el Cuadro 2 especificamos los aprendizajes esperados relacionados con este tema.

Cuadro 2. Aprendizajes esperados sobre las MTC para la Educación Primaria en México (SEP, 2017)

\begin{tabular}{|c|l|}
\hline Grado & \multicolumn{1}{|c|}{ Aprendizaje esperado } \\
\hline $4^{\circ}$ & Uso e interpreta la moda de un conjunto de datos (p. 319). \\
\hline $5^{\circ}$ & Interpreta la moda (p. 320). \\
\hline $6^{\circ}$ & $\begin{array}{l}\text { Usa e interpreta la moda, la media aritmética y el rango de un conjunto de datos (p. } \\
321) .\end{array}$ \\
\hline
\end{tabular}

Fuente: elaborado por los autores

En ambos casos se observa la importancia que tienen las MTC y que deben ser enseñadas en los últimos tres años de la Educación Primaria. Para este trabajo, no consideramos las últimas modificaciones curriculares, por estar próximas a implementarse. 


\section{Antecedentes}

\section{Investigaciones sobre MTC}

Algunas investigaciones acerca de las MTC se han centrado, mayoritariamente, en la media aritmética (Batanero, Godino y Navas, 1997; Estrella, 2016; Garrett y García, 2008; Ortiz, Font y Mayén, 2009; Marques, Guimarães y Gitinara, 2011); otras combinan dos o más de ellas (Escobedo y Mayén, 2018; Estrella, 2008; Neres y Cantanhêde, 2018; Rodríguez-Alveal, DíazLevicoy y Maldonado-Fuentes, 2018; Rodríguez-Alveal, Maldonado-Fuentes y Sandoval, 2012); y son pocas las que indagan exclusivamente la mediana o la moda (e.g., Mayén y Batanero, 2009).

Sobre la media aritmética, Batanero et al. (1997) estudian su comprensión por 273 estudiantes de la Facultad de Ciencias de la Educación. Los resultados evidencian la existencia de errores conceptuales y las dificultades en la aplicación de los conocimientos sobre este objeto estadístico, en especial en el tratamiento de los valores atípicos, por lo que señalan la necesidad de potenciar los contenidos estadísticos en la formación de profesores. En esta misma línea, Ortiz et al. (2009) indagan los significados personales de futuros profesores de Educación Primaria, observando dificultades relacionadas con la comprensión de la media y sus propiedades.

Garrett y García (2008) analizan los niveles de comprensión de la media aritmética en estudiantes de secundaria (130) y universitarios (97). Los resultados muestran que los estudiantes: 1) no tienen noción del valor atípico; 2) no consideran la media aritmética como mejor estimador, sino que otorgan esta propiedad a la moda; 3) no son conscientes de la influencia de los valores nulos; y 4) no comprenden ni usan las propiedades significativas de la media aritmética. Además, no se observan diferencias importantes entre ambos grupos de estudiantes.

Por su parte, Marques et al. (2011) analizan la comprensión que alcanzan profesores y estudiantes de Educación Primaria sobre la media aritmética, por medio de la aplicación de dos cuestionarios paralelos, donde variaba la representación entre gráfico de barras y el texto. Los resultados muestran que la comprensión de la media se ve afectada por la representación usada (gráfico o texto); además se observó, en ambos grupos, la dificultad para trabajar con esta MTC, aunque los profesores obtuvieron mejores resultados. 


\section{Investigaciones sobre las MTC en libros de texto}

La importancia de investigar libros de texto radica en que cumple un papel transcendental en las decisiones didácticas que toma el profesor, principalmente porque cualquier tema que se enseñará se hará según el contexto, dificultad y modalidad que determine el texto (Ortiz, 2002). Además, "si en un texto aparece un significado sesgado, este puede llegar a transmitirse a los alumnos, debiendo el profesor que los usa mantener una permanente vigilancia epistemológica sobre el contenido" (Op. cit., p. 13).

En Cobo y Batanero (2004) se presenta una caracterización del significado institucional que otorgan los libros de texto de Educación Secundaria Obligatoria de España respecto al concepto de media aritmética. Los resultados evidencian el uso de variedad de formas de presentar el concepto, mientras que el trabajo se centra en la memorización de definiciones y en el manejo de la formulas, dejando de lado el estudio de sus propiedades.

Cabrera (2014) estudió el tratamiento de la media aritmética en libros de texto de Educación Secundaria de Perú y su influencia en la comprensión por parte de 32 estudiantes de ingeniería. Para ello, se aplicó un cuestionario, diseñado a partir de los resultados del análisis de los textos. Los resultados muestran que las respuestas de los estudiantes están relacionadas con lo señalado en los textos, especialmente las definiciones, el uso de su algoritmo y aspectos de memorización.

Por su parte, Anjos y Gitirana (2008) estudian el concepto de media aritmética en libros de texto de Educación Primaria en Brasil. Los resultados muestran que el trabajo con este objeto estadístico es, según las propiedades descritas por Strauss y Bichler (1988), en algunos aspectos, óptimo y, en otros insuficientes. Destacando, en el primer caso, que cerca de $75 \%$ de las actividades consideran una media aritmética no coincide con algún valor del conjunto. Entre las propiedades poco exploradas se tienen: la media aritmética está comprendida entre los valores extremos de la distribución y la suma de las desviaciones de los datos respecto de la media es cero.

Asimismo, Carvalho y Gitirana (2014) analizan el tratamiento de la media aritmética en libros de texto en Brasil, observando que la mayoría de las actividades (63\%) no promueven la reflexión de sus propiedades propuestas por Strauss y Bichler (1988), el significado explotado mayoritariamente es considerarlo como un elemento representativo de un grupo $(86,8 \%)$ y la representación más usada en la media aritmética es la lengua materna (56\%). 
Estrella (2008) estudia la transposición didáctica de las MTC en un libro de texto de $7^{\circ}$ de Educación Primaria en Chile. La autora concluye que el texto carece de un lenguaje matemático fino, presenta ambigüedad en el uso de los términos y sus significados, los conceptos son presentados por medio de fórmulas y las actividades se reducen a su aplicación inmediata. Por su parte, Ocoró y Ocoró (2016) analizan las actividades propuestas para la enseñanza de las MTC en dos libros de texto de $7^{\circ}$ grado en Colombia. Los resultados permiten detectar que no se presentan todas las propiedades de las MTC, no se trabaja la media ponderada y es poca la presencia de ejemplos que permitan ilustrar cómo aplicar cada MTC.

En general, las investigaciones aquí señaladas se han centrado en el análisis de la media aritmética, siendo escasas las enfocadas en las otras MTC. Bajo esta perspectiva, este trabajo aporta elementos de análisis y resultados enfocados en las tres MTC más importantes: media aritmética, mediana y moda.

\section{Método}

Para realizar el análisis de las actividades relacionadas con las MTC seguimos una metodología cualitativa (Pérez-Serrano, 1994), con un nivel de estudio descriptivo (Hernández, Fernández y Baptista, 2010) y mediante el análisis de contenido (López-Noguero, 2002). Se utilizó una muestra no probabilística del tipo intencional (Hernández, Fernández y Baptista, 2010), donde consideramos los libros de texto por su amplia cobertura, uso y prestigio en la enseñanza mexicana para $4^{\circ}$ a $6^{\circ}$ curso de Educación Primaria en el área de matemática de la SEP y de la editorial privada Santillana. Las referencias de estos textos se observan en el Cuadro 3, los que se codificaron en T1, T2, T3, T4, T5 y T6 para facilitar su citación a lo largo del escrito. 
Cuadro 3. Datos de los libros de texto analizados

\begin{tabular}{|c|l|l|l|}
\hline Código & \multicolumn{1}{|c|}{ Autores } & \multicolumn{1}{|c|}{ Título } & Editorial \\
\hline T1 & SEP (2014a) & $\begin{array}{l}\text { Desafíos Matemáticos. Libro para el alumno. } \\
\text { Cuarto grado }\end{array}$ & SEP \\
\hline T2 & SEP (2014b) & $\begin{array}{l}\text { Desafíos Matemáticos. Libro para el alumno. } \\
\text { Quinto grado }\end{array}$ & SEP \\
\hline T3 & SEP (2014c) & $\begin{array}{l}\text { Desafíos Matemáticos. Libro para el alumno. } \\
\text { Sexto grado }\end{array}$ & SEP \\
\hline T4 & Cingerli et al. (2015) & $\begin{array}{l}\text { La Guía Santillana 4. Actividades para } \\
\text { aprender, convivir y ser }\end{array}$ & Santillana \\
\hline T5 & Cerón et al. (2015) & $\begin{array}{l}\text { La Guía Santillana 5. Actividades para } \\
\text { aprender, convivir y ser }\end{array}$ & Santillana \\
\hline T6 & Cerón et al. (2015) & $\begin{array}{l}\text { La Guía Santillana 6. Actividades para } \\
\text { aprender, convivir y ser }\end{array}$ & Santillana \\
\hline
\end{tabular}

Fuente: elaborado por los autores

Para el análisis de cada una de las actividades en los libros de texto se consideraron las siguientes variables:

- Tipo de MTC. Se identifica la (s) MTC que interviene (n) en la actividad: 1) media; 2) mediana; y 3) moda.

- Tipo de tarea. Es relacionada con lo que debe realizar el estudiante, de acuerdo a los datos entregados, en cada actividad. En ella se distinguen: 1) calcular una MTC; 2) calcular un dato dada una MTC; y 3) explicar.

- Representación. Está asociada con la forma en que se presentan los datos necesarios para desarrollar la actividad relacionada con las MTC: 1) listado de datos; 2) tabla de datos; 3) tabla de frecuencias; 4) tabla de doble entrada; y 5) gráfico de barras.

- Contexto. Corresponde al tipo de situación en que se enmarca la actividad, donde se presentan los datos y para los que tienen sentido el resultado obtenido; en este caso, consideramos los contextos descritos en PISA (OCDE, 2013): 1) personal; 2) profesional; 3) social; y 4) científico.

- Forma de trabajo. Relacionada con la organización de los estudiantes en el salón de clases para el desarrollo de las actividades, es decir, se indica si estos deben trabajar solos o por equipo, lo que se observa en el enunciado de las instrucciones de la actividad: 1) individual; y 2) grupal. 
Cada actividad del libro de texto, en que se hace referencia a una MTC, se ha clasificado de acuerdo a las unidades de análisis y categorías mencionadas. En algunos casos, como en el tipo de MTC y de tarea, en una misma actividad se pueden observar varias categorías, por lo que se contabilizará en cada categoría, según corresponda.

\section{Resultados}

La distribución de las actividades sobre MTC encontradas y analizadas en los libros de texto mexicanos de matemática para la Educación Primaria (editados para la SEP y de la editorial Santillana), según curso y editorial, se muestra en la Tabla 1. En ella observamos que la cantidad de actividades, en general, es escasa (tan solo 26 actividades en total). Situación que es similar si se analiza por curso, ya que en la mayoría de ellos hay 3 o 4 actividades; solo en el texto de la SEP de $6^{\circ}$ grado existen 8 actividades. De los tres cursos analizados, en $6^{\circ}$ es donde se concentra la mayor proporción de actividades (46,2\%); especialmente porque en el texto de la SEP se presenta una mayor cantidad de actividades $(57,1 \%$ para dicha editorial).

Tabla 1. Frecuencia (y porcentaje) de actividades analizadas por editorial

\begin{tabular}{cccc} 
Curso & SEP & Santillana & Total \\
\hline $4^{\text {o }}$ & $3(21,4)$ & $4(33,3)$ & $7(26,9)$ \\
$5^{\circ}$ & $3(21,4)$ & $4(33,3)$ & $7(26,9)$ \\
$6^{\circ}$ & $8(57,1)$ & $4(33,3)$ & $12(46,2)$ \\
\hline Total & $14(100)$ & $12(100)$ & $26(100)$ \\
\hline
\end{tabular}

Fuente: elaborado por los autores

A continuación, presentamos resultados del análisis de los libros de texto, tomando en cuenta las variables o unidades de análisis.

\section{Tipo de MTC}

La primera variable considera en este estudio es el tipo de MTC que se trabaja en la actividad. La moda se observa, por ejemplo, en la actividad de la Figura 1, donde el estudiante debe obtener esta MTC de las calificaciones de un bimestre de dos niños (Jesús y Mariano). 
Figura 1. Actividad sobre moda

En equipos de tres, resuelvan los problemas.

1. Estas son las calificaciones del tercer bimestre de Jesús y de Mariano:

\begin{tabular}{|c|c|c|c|}
\hline \multicolumn{2}{|c|}{ Alumno: Jesús Mena Rosas } & \multicolumn{2}{|c|}{ Alumno: Mariano Luna López } \\
\hline Español & 5 & Español & 7 \\
\hline Matemáticas & 7 & Matemáticas & 8 \\
\hline C. Naturales & 8 & C. Naturales & 9 \\
\hline Historia & 6 & Historia & 7 \\
\hline Geografía & 7 & Geografia & 10 \\
\hline F. Cívica y Ética & 7 & F. Cívica y Ética & 7 \\
\hline E. Fisica & 6 & E. Fisica & 8 \\
\hline E. Artistica & 7 & E. Artistica & 7 \\
\hline
\end{tabular}

a) ¿Cuál es la moda de las calificaciones de Mariano y cuál es la moda de las calificaciones de Jesús?

Fuente: T 1, p. 156

La media aritmética se puede observar en la actividad de la Figura 2, donde se pide calcular esta MTC a partir de un listado de datos, y mencionar el proceso seguido para obtener dicho estadígrafo.

Figura 2. Actividad sobre media

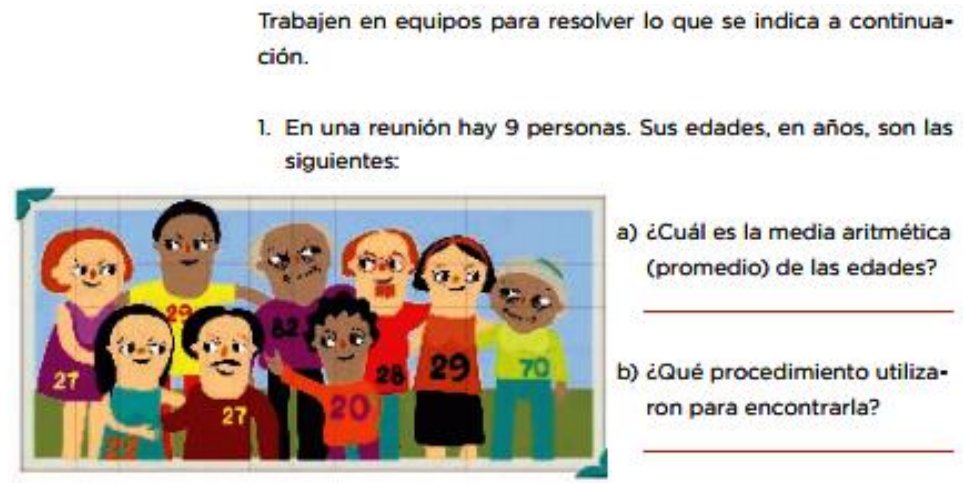

Fuente: T3, p. 104

La mediana se trabaja en la actividad de la Figura 3, que es continuación de la mostrada en la Figura 2. En ella se introduce la forma de calcular esta MTC cuando se tiene un listado de datos sin agrupar. 
Figura 3. Actividad sobre mediana

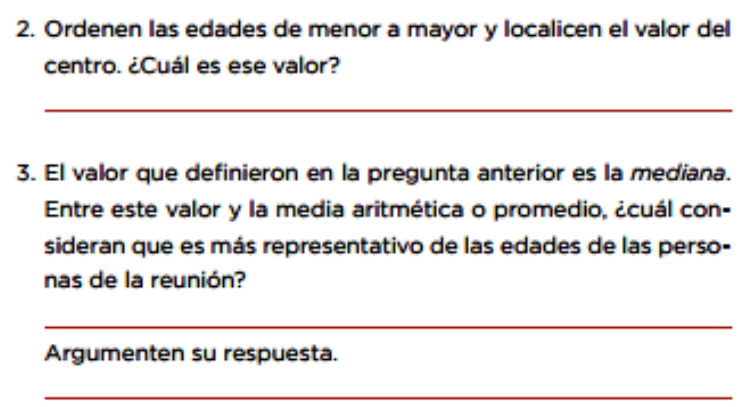

Fuente: T3, p. 104

En la Tabla 2 se presenta la distribución de las MTC en los libros de texto, según la editorial y el curso. Observamos que la moda se comienza a trabajar en $4^{\circ}$ grado, en ambos textos, sin la presencia de las otras dos MTC. En $5^{\circ}$ curso, junto a la moda, se introducen actividades sobre la media aritmética. Finalmente, en $6^{\circ}$ grado se presentan actividades para trabajar la mediana, y se mantiene el trabajo con la moda y media.

En general, las actividades suelen considerar más de una MTC a la vez, es decir, los datos de una misma situación se utilizan para trabajar una o más MTC. Por ejemplo, en la actividad de la Figura 2 y 3 se trabaja la media y la mediana con los mismos datos. Esta situación influye en el predominio de la media y moda, ambas presentes en un 65,4\% de las actividades.

Tabla 2. Frecuencia (y porcentaje) de MTC encontrada en las actividades por curso y editorial

\begin{tabular}{|c|c|c|c|c|c|c|c|c|c|}
\hline \multirow{2}{*}{ MTC } & \multicolumn{4}{|c|}{ SEP } & \multicolumn{4}{|c|}{ Santillana } & \multirow{2}{*}{ Total } \\
\hline & $4^{\circ}$ & $5^{\circ}$ & $6^{\circ}$ & Total & $4^{\circ}$ & $5^{\circ}$ & 6 & Total & \\
\hline Media & $0(0)$ & $3(100)$ & $6(75)$ & $9(64,3)$ & $0(0)$ & $4(100)$ & $4(100)$ & $8(66,7)$ & $17(65,4)$ \\
\hline Mediana & $0(0)$ & $0(0)$ & $7(87,5)$ & $7(50)$ & $0(0)$ & $0(0)$ & $4(100)$ & $4(33,3)$ & $11(42,3)$ \\
\hline Moda & $3(100)$ & $1(33,3)$ & $4(50)$ & $8(57,1)$ & $4(100)$ & $4(100)$ & $1(25)$ & $9(75)$ & $17(65,4)$ \\
\hline Total & $3(100)$ & $3(100)$ & $8(100)$ & $14(100)$ & $4(100)$ & $4(100)$ & $4(100)$ & $12(100)$ & $26(100)$ \\
\hline
\end{tabular}

Fuente: elaborado por los autores

\section{Tipo de tarea}

En segundo lugar, consideramos la variable tipo de tarea, es decir, lo que se pide realizar a los estudiantes. Una de las categorías encontradas es calcular MTC, que consiste en obtener, de acuerdo a lo indicado en el enunciado de la actividad, la media, mediana y/o moda, con los datos o información proporcionada. Un ejemplo de esta tarea se presenta en la Figura 4, donde 
junto con completar una tabla con algunos datos de familiares (madre, padre y abuelas), se solicita calcular las tres MTC.

Figura 4. Actividad de calcular MTC

Completa la tabla y calcula la media, la moda y la mediana de las dos columnas.

\begin{tabular}{|c|l|l|}
\hline \multicolumn{1}{|c|}{ Familiar } & Edad & Años de estudio \\
\hline Papá & \\
\hline Mamá & \\
\hline Abuela paterna & Media de años de estudio: \\
\hline Abuela materna & Mediana de años de estudio: \\
\hline Media de edad: & Moda de años de estudio: \\
\hline Mediana de edad: \\
\hline
\end{tabular}

Fuente: T6, p. 225

Otra tarea que hemos identificado es calcular un dato, que consiste en obtener uno de los valores del conjunto de datos original dada una MTC. Por ejemplo, en el apartado 2 de la actividad de la Figura 5, vemos que los estudiantes deben calcular la calificación mínima que deben obtener Ernesto, Joaquín, Sara y Elisa para acceder a una beca, es decir, lograr una media de 8,2 .

Figura 5. Actividad de calcular un dato

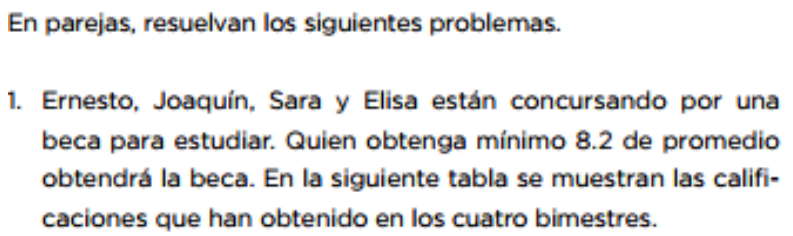

\begin{tabular}{|c|c|c|c|c|c|} 
& \multicolumn{2}{|c|}{$\begin{array}{c}\text { Primer } \\
\text { bimestre }\end{array}$} & $\begin{array}{c}\text { Segundo } \\
\text { bimestre }\end{array}$ & $\begin{array}{c}\text { Tercer } \\
\text { bimestre }\end{array}$ & \multicolumn{2}{c|}{$\begin{array}{c}\text { Cuarto } \\
\text { bimestre }\end{array}$} & $\begin{array}{c}\text { Quinto } \\
\text { bimestre }\end{array}$ \\
\hline Ernesto & 7 & 8 & 8 & 8 & \\
\hline Joaquín & 8 & 7 & 8 & 9 & \\
\hline Sara & 8 & 9 & 8 & 8 & \\
\hline Elisa & 7 & 8 & 8 & 9 & \\
\hline
\end{tabular}

\footnotetext{
a) Hasta el cuarto bimestre, ¿quién tiene posibilidades de obtener la beca?
}

b) ¿Qué calificación como mínimo necesita obtener cada uno en el quinto bimestre para que le den la beca?

Fuente: T2, p. 189 
Explicar, es el tercer tipo de tarea encontrada, consiste en describir detalladamente algún proceso seguido, entregar comentarios o justificaciones sobre determinado resultado, beneficio, desventaja o elección de una MTC. Un ejemplo de esta tarea se presenta en la pregunta 3 de la actividad de la Figura 3, donde se debe argumentar sobre la representatividad de la media o mediana frente a una determinada situación.

A modo de resumen, en la Tabla 3 mostramos la distribución de las tareas presenten en los libros de texto analizados. En ella, observamos que se hace un mayor énfasis a los procesos algorítmicos para obtener los valores de la MTC, calcular MTC, presente en todos los libros y cursos considerados, abarcando un 92,3\%. Le sigue, en un segundo lugar, la tarea de explicar en un 50\%, presente en los tres libros de la SEP $(78,6 \%)$ y sólo en $5^{\circ}$ curso de Santillana (50\%). La tarea más escasa, que se presenta sólo una vez en el libro de $5^{\circ}$ de Educación Primaria de la $\operatorname{SEP}(33,3 \%)$, es calcular un dato.

Tabla 3. Frecuencia (y porcentaje) del tipo de tarea pedida en las actividades por curso y editorial

\begin{tabular}{lcccccccccc}
\hline \multirow{2}{*}{ Tarea } & \multicolumn{9}{c}{ SEP } & \multicolumn{5}{c}{ Santillana } & \multirow{2}{*}{ Total } \\
\cline { 2 - 7 } Calcular MTC & $4^{\circ}$ & $5^{\circ}$ & $6^{\circ}$ & Total & $4^{\circ}$ & $5^{\circ}$ & 6 & Total & \\
Calcular un dato & $0(0)$ & $1(33,3)$ & $0(0)$ & $1(7,1)$ & $0(0)$ & $0(0)$ & $0(0)$ & $0(0)$ & $1(3,8)$ \\
Explicar & $2(66,7)$ & $2(66,7)$ & $7(87,5)$ & $11(78,6)$ & $0(0)$ & $2(50)$ & $0(0)$ & $2(16,7)$ & $13(50)$ \\
\hline
\end{tabular}

Fuente: elaborado por los autores

\section{Representación}

Esta variable está relacionada con los objetos estadísticos que intervienen en una actividad sobre MTC, concretamente, sobre la forma en que se proporcionan los datos. El primero de ellos, es el listado de datos, donde no se utiliza la idea de frecuencia y distribución. Por ejemplo, en los datos de la Figura 2, donde se entregan los datos uno a uno, sin agrupar los iguales y calcular las frecuencias.

Una segunda forma de representación corresponde a la tabla de datos, es decir, es una tabla simple, donde no se trabaja la idea de frecuencia ni de distribución, sino sólo la idea de variable y valor (Díaz-Levicoy, Morales y Vásquez, 2017; Lahanier-Reuter, 2003) y observada en actividades de libros de texto de Educación Primaria (Díaz-Levicoy, Morales y LópezMartín, 2015; Díaz-Levicoy, Ruz y Molina-Portillo, 2017). Este tipo de tabla la vemos 
ejemplificada en la actividad de la Figura 4, donde el estudiante debe registrar datos particulares, que no provienen del cálculo de frecuencias.

Una tercera forma de representación es la tabla de frecuencias, en la que se representa la repetición (frecuencias) de aparición de los valores o categorías de las variables (DíazLevicoy, Morales et al., 2017; Lahanier-Reuter, 2003); también observada en libros de texto de Educación Primaria (Díaz-Levicoy et al, 2015; Díaz-Levicoy, Ruz et al., 2017). Un ejemplo de esta representación se presenta en la actividad de la Figura 6, donde se han registrado las frecuencias de la cantidad de veces que se han vendido uniformes escolares según su talla; con lo que se trabaja la MTC de la moda.

Figura 6. Actividad de calcular un dato

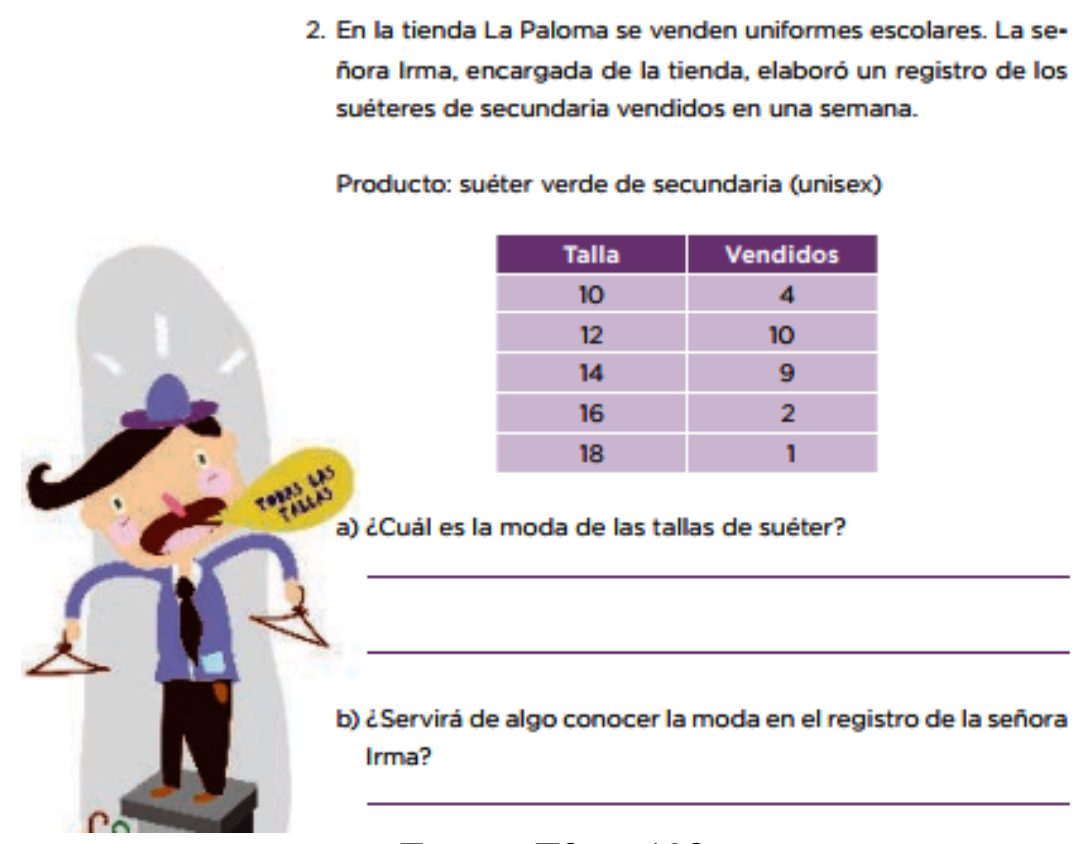

Fuente: T2, p. 198

En la Figura 7 se muestra un ejemplo de la cuarta forma de representación encontrada, tabla de doble entrada, que se caracteriza por cruzar dos variables; es decir, un valor está relacionado con dos categorías de una variable de modo simultáneo. En este caso, se muestra la frecuencia de estudiantes, por ciclo y total, de los juegos que les gusta más, donde las variables son juegos favoritos y ciclo al que pertenecen los alumnos encuestados. 
Figura 7. Actividad de calcular un dato

En la escuela se realizó una encuesta sobre el juego favorito de los alumnos.

\begin{tabular}{|l|c|c|c|c|}
\hline \multirow{2}{*}{ Juego favorito } & \multicolumn{4}{|c|}{ Número de alumnos } \\
\cline { 2 - 5 } & Primer ciclo & Segundo Ciclo & Tercer ciclo & Total \\
\hline Brincar la cuerda & 10 & 12 & 15 & \\
\hline Basta & & 16 & 10 & 40 \\
\hline Avión & 7 & 9 & 25 & \\
\hline Encantados & 12 & 7 & & 37 \\
\hline Víbora de la mar & 23 & 4 & 3 & \\
\hline Otros & 12 & & 15 & 38 \\
\hline Total & & 59 & 86 & \\
\hline
\end{tabular}

De acuerdo con la información anterior, contesta las preguntas.

¿En qué ciclo tienen mayor preferencia por jugar Basta?

¿En qué ciclo se juega más Encantados?

En el primer ciclo, ¿cuál es el juego que más gusta?

¿Qué juego gusta más en toda la escuela?

Fuente: T4, p. 367

Finalmente, la quinta representación utilizada para trabajar las actividades sobre las MTC es el gráfico de barras, que se define como una "representación gráfica que puede ser usada para representar la distribución de frecuencia de variables cualitativas, cuantitativas discretas o incluso variables continuas, si han sido discretizadas y diferentes intervalos de valores se han transformado en categorías" (Arteaga, 2011, p. 8). Por ejemplo, en la Figura 8 vemos que se ha utilizado un gráfico de barras para presentar las frecuencias del tiempo de caída de un balón.

Figura 8. Gráfico de barras como tipo de representación

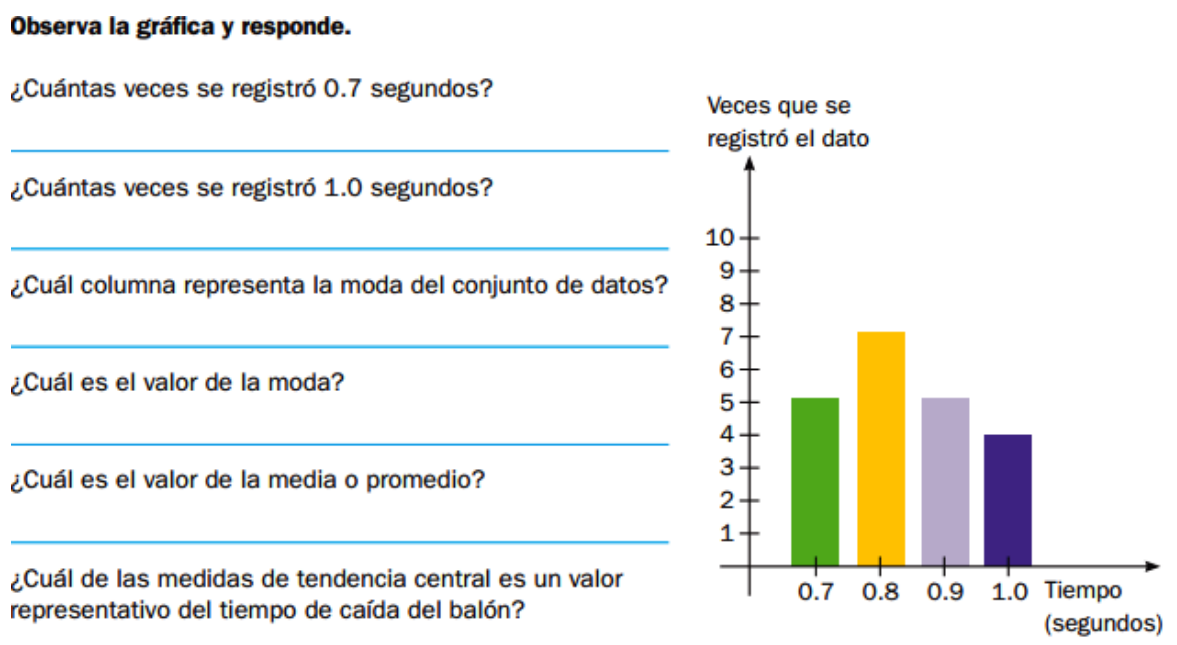

Fuente: T5, p. 381 
En la Tabla 4 resumimos la distribución de las representaciones utilizadas en los libros de texto para el trabajo con las MTC. A modo general, vemos el predominio del listado de datos como forma de representación para entregar los datos/información necesarios para resolver la actividad (38,5\%); aunque el 57,7\% utiliza alguna forma de representación tabular (tabla de datos, frecuencias o doble entrada), donde se destacan las tablas de datos presentes en el 34,6\% de las actividades. Ninguna de las representaciones se observa en todos los cursos y libros de texto analizados. No obstante, el listado de datos se presenta en los textos de todos los cursos de la SEP, y en $4^{\circ}$ y $5^{\circ}$ grado de Santillana; la tabla de datos en los tres libros SEP y únicamente en el texto de $6^{\circ}$ grado de Santillana; y las tablas de frecuencias aparecen en $4^{\circ}$ y $6^{\circ}$ de $\operatorname{los}$ libros de SEP y en sólo un libro de Santillana $\left(4^{\circ}\right)$. La tabla de doble entrada y el gráfico de barras se observan sólo una vez en las actividades para trabajar las MTC, en los textos de $4^{\circ}$ y $5^{\circ}$ curso de Santillana, respectivamente.

Tabla 4. Frecuencia (y porcentaje) del tipo de representación estadística ocupada en la actividad

\begin{tabular}{|c|c|c|c|c|c|c|c|c|c|}
\hline \multirow{2}{*}{ Representación } & \multicolumn{4}{|c|}{ SEP } & \multicolumn{4}{|c|}{ Santillana } & \multirow{2}{*}{ Total } \\
\hline & $4^{\circ}$ & $5^{\circ}$ & $6^{\circ}$ & Total & $4^{\circ}$ & $5^{\circ}$ & 6 & Total & \\
\hline Listado de datos & $1(33,3)$ & $1(33,3)$ & $3(37,5)$ & $5(35,7)$ & $2(50)$ & $3(75)$ & $0(0)$ & $5(41,7)$ & $10(38,5)$ \\
\hline Tabla de datos & $1(33,3)$ & $2(66,7)$ & $2(25)$ & $5(35,7)$ & $0(0)$ & $0(0)$ & $4(100)$ & $4(33,3)$ & $9(34,6)$ \\
\hline Tabla de frecuencia & $1(33,3)$ & $0(0)$ & $3(37,5)$ & $4(28,6)$ & $1(25)$ & $0(0)$ & $0(0)$ & $1(8,3)$ & $5(19,2)$ \\
\hline $\begin{array}{lll}\text { Tabla de } & \text { doble } \\
\text { entrada } & & \end{array}$ & $0(0)$ & $0(0)$ & $0(0)$ & $0(0)$ & $1(25)$ & $0(0)$ & $0(0)$ & $1(8,3)$ & $1(3,8)$ \\
\hline Gráfico de barras & $0(0)$ & $0(0)$ & $0(0)$ & $0(0)$ & $0(0)$ & $1(25)$ & $0(0)$ & $1(8,3)$ & $1(3,8)$ \\
\hline Total & $3(100)$ & $3(100)$ & $8(100)$ & $14(100)$ & $4(100)$ & $4(100)$ & $4(100)$ & $12(100)$ & $26(100)$ \\
\hline
\end{tabular}

Fuente: elaborado por los autores

\section{Contexto}

Otra de las unidades de análisis de interés en nuestro estudio es el contexto en el que se presentan las actividades, es decir, donde se presentan los datos y para los que tienen sentido el resultado obtenido. En este caso, consideramos los descritos en PISA (OCDE, 2013): 1) personal; 2) profesional; 3) social; y 4) científico. Esta ha sido una variable considerada en diferentes estudios (e.g., Díaz-Levicoy et al., 2015, 2018; Mingorance, 2014).

En el contexto personal, la situación se centra en actividades propias del niño, familia o pares (OCDE, 2013), lo observamos en la actividad de la Figura 4, donde se trabaja con datos 
de algunos familiares del estudiante, o en la Figura 7, donde se aborda una situación sobre los juegos favoritos de un grupo de niños. De igual modo, tiene cabida la actividad de la Figura 8 , donde se registra el tiempo de caída de un balón y que se corresponde a una actividad de juego.

El contexto profesional se centra en el mundo del trabajo (cálculo de coste, control de calidad, inventarios, toma de decisiones, entre otros). Ejemplo de este contexto lo vemos en la actividad de la Figura 6, donde se realiza un registro de las ventas de uniformes escolares de una tienda (La Paloma) en una semana.

El contexto social está relacionado con la comunidad local, nacional o mundial y “pueden involucrar (pero no están limitadas a) cosas como los sistemas de votación, el transporte público, el gobierno, las políticas públicas, la demografía, la publicidad, las estadísticas nacionales y la economía" (OCDE, 2013, p. 37). Ejemplo de este contexto lo observamos en la Figura 9, donde se presentan estadísticas nacionales sobre la población mexicana, aspectos demográficos, y donde se plantea la actividad: "De este conjunto de datos, ¿Será más representativa la moda, la mediana o la media aritmética? ¿Por qué?” (T3, p. 108)

Figura 9. Contexto social

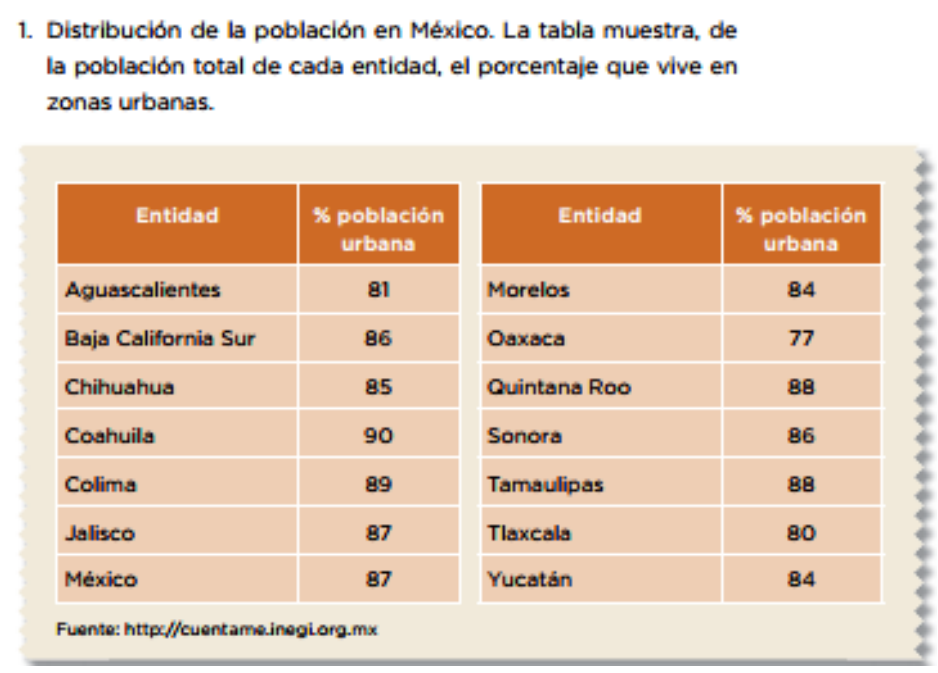

Fuente: T3, p. 107

Finalmente, el contexto científico, relacionado a la aplicación de la matemática a la naturaleza, ciencias y/o tecnología. Un ejemplo de este contexto se presenta en la situación descrita en la Figura 10, en ella se muestra el registro de las temperaturas entre las 8 de la mañana y las 8 de la noche en una población de la ciudad de Puebla. Sobre esta información, se pide calcular la moda y la media. 
Figura 10. Contexto científico

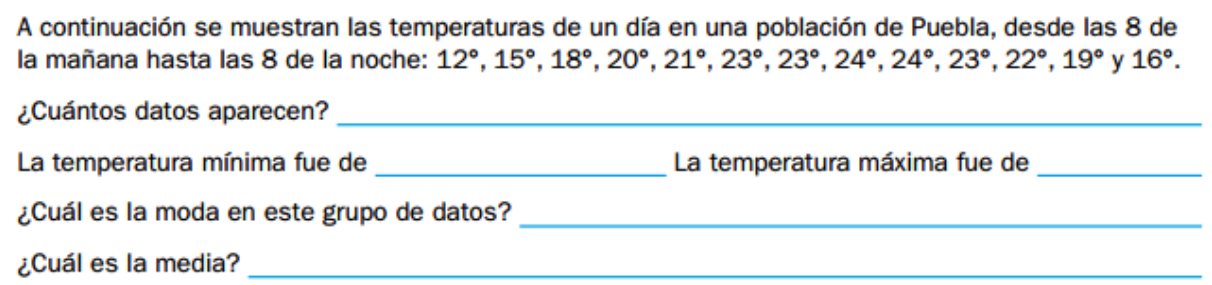

Fuente: T5, p. 381

En general, en la Tabla 5 observamos que predomina el contexto personal, presente en un $73,1 \%$ de las actividades y en todos los libros de texto analizado. Los contextos social y laboral se presentan esporádicamente (11,5\%), el primero sólo en $6^{\circ}$ curso y el segundo en $4^{\circ}$ y $5^{\circ}$ en los libros de la SEP, respectivamente. El contexto científico sólo se observa en el texto de $5^{\circ}$ grado de Santillana $(3,8 \%)$.

Tabla 5. Frecuencia (y porcentaje) del contexto utilizado en las actividades

\begin{tabular}{|c|c|c|c|c|c|c|c|c|c|}
\hline \multirow{2}{*}{ Contexto } & \multicolumn{4}{|c|}{ SEP } & \multicolumn{4}{|c|}{ Santillana } & \multirow{2}{*}{ Total } \\
\hline & $4^{\circ}$ & $5^{\circ}$ & $6^{\circ}$ & Total & $4^{\circ}$ & $5^{\circ}$ & 6 & Total & \\
\hline Personal & $1(33,3)$ & $2(66,7)$ & $5(62,5)$ & $8(57,1)$ & $4(100)$ & $3(75)$ & $4(100)$ & $11(91,7)$ & $19(73,1)$ \\
\hline Social & $0(0)$ & $0(0)$ & $3(37,5)$ & $3(21,4)$ & $0(0)$ & $0(0)$ & $0(0)$ & $0(0)$ & $3(11,5)$ \\
\hline Laboral & $2(66,7)$ & $1(33,3)$ & $0(0)$ & $3(21,4)$ & $0(0)$ & $0(0)$ & $0(0)$ & $0(0)$ & $3(11,5)$ \\
\hline Científico & $0(0)$ & $0(0)$ & $0(0)$ & $0(0)$ & $0(0)$ & $1(25)$ & $0(0)$ & $1(8,3)$ & $1(3,8)$ \\
\hline Total & $3(100)$ & $3(100)$ & $8(100)$ & $14(100)$ & $4(100)$ & $4(100)$ & $4(100)$ & $12(100)$ & $26(100)$ \\
\hline
\end{tabular}

Fuente: elaborado por los autores

\section{Forma de trabajo}

La última unidad de análisis considerada es forma de trabajo que se infiere de las instrucciones y enunciado de las actividades, las que pueden ser individuales o en grupales (en equipo) y que han sido considerados en estudios previos (e.g., Díaz-Levicoy et al., 2018). Ejemplos de las actividades individuales se observan, de acuerdo a la redacción de los enunciados, en la Figura 4: "completa la tabla y calcula la media, la moda y la mediana de las dos columnas” (T6, p. 225), Figura 8: “observa la gráfica y responde” (T5, p. 107). De modo similar, actividades que se sugieren para trabajar en equipo podemos ver en la Figura 1: “en equipos de tres, resuelvan los problemas" (T1, p. 156), la Figura 2: "trabajen en equipos para 
resolver lo que se indica a continuación" (T3, p. 104), o la Figura 5: "En parejas, resuelvan los siguientes problemas" (T2, p. 189).

En la Tabla 6 presentamos la distribución de las actividades según la forma de trabajo en que se sugieren ser abordadas. El 53,8\% de las actividades se plantean para ser resueltas en grupo y en su totalidad corresponden a los libros de la SEP. El porcentaje restante (46,2\%) corresponde a aquellas que se proponen para ser desarrolladas en forma individual, presentes exclusivamente en los textos de Santillana.

Tabla 6. Frecuencia (y porcentaje) de la forma de trabajo en que se plantean las actividades

\begin{tabular}{|c|c|c|c|c|c|c|c|c|c|}
\hline \multirow{2}{*}{$\begin{array}{c}\text { Forma de } \\
\text { trabajo }\end{array}$} & \multicolumn{4}{|c|}{ SEP } & \multicolumn{4}{|c|}{ Santillana } & \multirow[b]{2}{*}{ Total } \\
\hline & $4^{\circ}$ & $5^{\circ}$ & $6^{\circ}$ & Total & $4^{\circ}$ & $5^{\circ}$ & 6 & Total & \\
\hline Individual & $0(0)$ & $0(0)$ & $0(0)$ & $0(0)$ & $4(100)$ & $4(100)$ & $4(100)$ & $12(100)$ & $12(46,2)$ \\
\hline Grupal & $3(100)$ & $3(100)$ & $8(100)$ & $14(100)$ & $0(0)$ & $0(0)$ & $0(0)$ & $0(0)$ & $14(53,8)$ \\
\hline Total & $3(100)$ & $3(100)$ & $8(100)$ & $14(100)$ & $4(100)$ & $4(100)$ & $4(100)$ & $12(100)$ & $26(100)$ \\
\hline
\end{tabular}
Fuente: elaborado por los autores

\section{Conclusiones}

En este trabajo presentamos el análisis de las actividades relacionadas con las MTC en libros de texto de la SEP y de la editorial privada Santillana de $4^{\circ}$ a $6^{\circ}$ grado de la Educación Primaria en México. El análisis se realizó tomando en cuenta el tipo de MTC, el tipo de tarea, la representación, el contexto y la forma de trabajo. Se identificaron en total 26 actividades, 14 en los textos de la SEP y 12 en los de Santillana.

Respecto al tipo de $M T C$, identificamos que la moda se introduce en $4^{\circ}$ grado, la media en $5^{\circ}$ y la mediana en $6^{\circ}$, en concordancia con lo explicitado en las directrices curriculares (SEP, 2011b, 2011c, 2011d). Además, las MTC de moda y media aritmética son las que están presentes en la mayoría de las actividades encontradas $(65,4 \%)$.

Por otra parte, para la unidad de análisis tipo de tarea encontramos que el 92,3\% de las actividades piden el desarrollar algún proceso matemático sencillo (calcular MTC). Esto coincide con lo reportado por otras investigaciones, donde se observa un predominio aspectos procedimentales de las MTC (Cabrera, 2014; Cobo y Batanero, 2004; Estrella, 2008).

El listado de datos $(38,5 \%)$ y las tablas (de datos, frecuencia y doble entrada) $(57,7 \%)$ son las presentaciones más utilizadas para trabajar con las MTC. El gráfico de barras sólo se observa en una actividad de $5^{\circ}$ de Educación Primaria, este resultado nos preocupa, en especial 
por la importancia de los gráficos estadísticos en la sociedad y la utilidad para conectar diversos temas de matemática y estadística. Por lo que debería tener mayor preponderancia.

Respecto del contexto en que se presentan las situaciones en que se trabajan las MTC, vemos que predomina el personal $(73,1 \%)$, resultados que coinciden con trabajos previos sobre otros temas de estadística (e.g., Díaz-Levicoy, Ferrada, Salgado-Orellana y Vásquez, 2019; Díaz-Levicoy et al., 2015, 2018) o matemática (e.g., Álvarez y Blanco, 2015). Pese a ello, es necesario incluir, poco a poco, los otros tipos de contextos.

Sobre la forma de trabajo, vemos que la mayoría de las actividades se explicitan para ser trabajadas de manera de forma grupal $(53,8 \%)$, pero este resultado se debe a que los textos de la SEP presentan dos actividades más que los de Santillana. Lo anterior está dado porque todas las actividades de los textos de Santillana se sugieren trabajar en forma individual y los de la SEP en forma individual. En este sentido, el profesor sería el encargado de cambiar la forma de trabajo, generando instancias tanto individual como grupal.

Este estudio, pese a la limitación con el número de libros de texto analizados, nos permite tener una aproximación a la enseñanza de las MTC en el contexto mexicano, como así también observar la escasa relevancia que se le asigna a este tema en los libros de texto y la necesidad imperiosa de aumentar la cantidad de actividades, con mayor variedad de contexto, representaciones y forma de trabajar.

Este trabajo sugiere diferentes líneas de investigación futura, tales como indagar en la forma que este tema se trabaja en el aula de Educación Primaria, los niveles de comprensión que alcanzan los estudiantes, y si los futuros profesores de estos niveles tienen las competencias disciplinares y didácticas para su enseñanza.

\section{Referencias}

Álvarez, R. y Blanco, L. (2015). Evaluación en matemáticas: introducción al Álgebra y Ecuaciones en $1^{\circ}$ ESO. UNIÓN. Revista Iberoamericana de Educación Matemática, 42, 133-149.

Anjos, D. y Gitirana, V. (2008). Exploração do conceito de média nos em livros didáticos das séries finais do Ensino Fundamental. En V. Gitirana, F. Bellemain y V. Andrade (Eds.), Anais do $2^{\circ}$ Simpósio Internacional de Pesquisa em Educação Matemática (pp. 1-9). Recife: Universidad Federal de Pernambuco.

Arteaga, P. (2011). Evaluación de conocimientos sobre gráficos estadísticos y conocimientos didácticos de futuros profesores (Tesis Doctoral). Universidad de Granada, España. 
Arteaga, P., Batanero, C., Cañadas, G. y Contreras, J. M. (2011). Las tablas y gráficos estadísticos como objetos culturales. Números. Revista de Didáctica de las Matemáticas, $76,55-67$.

Batanero, C. (2001). Didáctica de la Estadística. Granada: Grupo de Investigación de Educación Estadística de la Universidad de Granada.

Batanero, C., Godino, J. D. y Navas, F. (1997). Concepciones de maestros de primaria en formación sobre los promedios. En H. Salmerón (Ed.), VII Jornadas LOGSE: Evaluación Educativa (pp. 310-324). Granada: Universidad de Granada.

Braga, G. y Belver, J. L. (2016). El análisis de libros de texto: una estrategia metodológica en la formación de los profesionales de la educación. Revista Complutense de Educación, 27(1), 199-218.

Cabrera, B. (2014). Contenido de la media aritmética en los libros de texto y su influencia en la comprensión por estudiantes del primer ciclo de la Universidad Nacional Micaela Bastidas de Apurímac. En N. Rubio (Ed.), VII Coloquio Internacional Enseñanza de las Matemáticas. Educación Matemática en contexto (pp. 1173-1175). Lima: Pontificia Universidad Católica del Perú.

Cantoral, R., Montiel, G. y Reyes-Gasperini, D. (2015). Análisis del discurso Matemático Escolar en los libros de texto, una mirada desde la Teoría Socioepestemológica. Avances de Investigación en Educación Matemática, 8, 9-28.

Carvalho, J. I. F. y Gitirana, V. (2014). Média aritmética - uma análise das atividades do livro didáctico de matemática adotados no brasil. En P. Lestón (Ed.), Acta Latinoamericana de Matemática Educativa (Vol. 27, pp. 681-688). Ciudad de México: Comité Latinoamericano de Matemática Educativa.

Cerón, F., García, S. D., Hernández, M. C., Macías, G., Maya, E. C., Puebla, J. J. y Rivera, M. (2015). La Guía Santillana 5. Actividades para aprender, convivir y ser. Ciudad de México: Santillana.

Cerón, F., García, S. D., Lugo, J. O., Martínez, M., Maya, E. C., Puebla, J. J., Rivera, M., Sanz, R. y Zeable, J. (2015). La Guía Santillana 6. Actividades para aprender, convivir y ser. Ciudad de México: Santillana.

Chevallard, Y. (1991). La transposición didáctica. Del saber sabio al saber enseñado. Buenos Aires: Aique.

Cingerli, B. S., Granja, E., Iturbe, B., Larios, C., Puebla, J. J., Olvera, Y. M., Ortega, M. T. y Osorio, I. (2015). La Guía Santillana 4. Actividades para aprender, convivir y ser. Ciudad de México: Santillana.

Cobo, B. (2003). Significado de las medidas de posición central para estudiantes de secundaria (Tesis doctoral). Universidad de Granada, España.

Cobo, B. y Batanero, C. (2004). Significado de la media en los libros de texto de secundaria. Enseñanza de las Ciencias, 22(1), 5-18.

Contreras, J. M. y Molina-Portillo, E. (2019). Elementos clave de la cultura estadística en el análisis de la información basada en datos. En J. M. Contreras, M. M. Gea, M. M. LópezMartín y E. Molina-Portillo (Eds.), Actas del Tercer Congreso Internacional Virtual de Educación Estadística (pp. 1-12). Granada: Universidad de Granada.

Del Pino, G. y Estrella, S. (2012). Educación estadística: Relaciones con la matemática. Pensamiento Educativo. Revista de Investigación Educacional Latinoamericana, 49(1), 53-64. 
Díaz-Levicoy, D., Ferrada, C., Salgado-Orellana, N. y Vásquez, C. (2019). Análisis de las actividades evaluativas sobre estadística y probabilidad en libros de texto chilenos de Educación Primaria. Premisa, 21(80), 5-21

Díaz-Levicoy, D., Giacomone, B. y Arteaga, P. (2017). Caracterización de los gráficos estadísticos en libros de texto argentinos del segundo ciclo de Educación Primaria. Profesorado. Revista de Currículum y Formación del Profesorado, 21(2), 299-326.

Díaz-Levicoy, D., Morales, R. y López-Martín, M. M. (2015). Tablas estadísticas en libros de texto chilenos de $1^{\circ}$ y $2^{\circ}$ año de Educación Primaria. Revista Paranaense de Educação Matemática, 4(7), 10-39.

Díaz-Levicoy, D., Morales, R. y Vásquez, C. (2017). Construcción de tablas estadísticas por estudiantes chilenos de tercero de Educación Primaria. Educação \& Linguagem, 20(1), 149-166.

Díaz-Levicoy, D., Ruz, F. y Molina-Portillo, E. (2017). Tablas estadísticas en libros de texto chilenos de tercer año de Educación Primaria. Espaço Plural, 18(36), 196-218.

Díaz-Levicoy, D., Vásquez, C. y Molina-Portillo, E. (2018). Estudio exploratorio sobre tablas estadísticas en libros de texto de tercer año de Educación Primaria. TANGRAM. Revista de Educação Matemática, 1(2), 18-39.

Escobedo, J. M. y Mayén, S. (2018). Evolución en la comprensión de estudiantes de telebachillerato de un problema de estimación de media y mediana a partir de un gráfico. En J. D. Zacarías, H. Cruz, F. Velasco, B. Juárez, V. H. Vázquez, H. Reyes y F. Tajonar (Eds.), Actualidad en la Educación Estadística y Probabilística (pp. 111-137). Puebla: Benemérita Universidad Autónoma de Puebla.

Estrella, S. (2008). Medidas de tendencia central en la enseñanza básica en Chile. Revista Chilena de Educación Matemática, 4(1), 20-32.

Estrella, S. (2016). Comprensión de la media por profesores de educación primaria en formación continua. REDIE. Revista Electrónica de Investigación Educativa, 18(1), 1-22.

Font, V. (2008). Enseñanza de la Matemática. Tendencias y perspectivas. En C. Gaita (Ed.), Actas III Coloquio Internacional sobre Enseñanza de las Matemáticas (pp. 21-64). Lima: PUCP.

Garrett, A. y García. J.A. (2008). Caracterización de la comprensión de algunos aspectos de la media aritmética: un estudio con alumnos de secundaria y universitarios. Enseñanza de la Matemática, 17(1), 31-57

Herbel, B. A. (2007). From intended curriculum to written curriculum: Examining the "voice" of a mathematics textbook. Journal for Research in Mathematics Education, 38(4), 344369.

Hernández, R., Fernández, C. y Baptista, P. (2010). Metodología de la investigación. México D.F.: McGraw Hill.

Lahanier-Reuter, D. (2003). Différents types de tableaux dans l'enseignement des statistiques. SPIRALE. Revue de Recherches en Éducation, 32, 143-154.

López-Noguero, F. (2002). El análisis de contenido como método de investigación. XXI. Revista de Educación, 4, 167-180.

Marques, M., Guimarães, G. y Gitirana, V. (2011). Compreensões de alunos e professores sobre média aritmética. BOLEMA. Boletim de Educação Matemática, 24(40), 725-745. 
Mayén, S. (2009). Comprensión de las medidas de tendencia central por estudiantes mexicanos de Educación Secundaria y Bachillerato (Tesis doctoral). Universidad de Granada, España.

Mayén, S. y Batanero, C. (2009). Conflictos semióticos en estudiantes mexicanos de bachillerato y secundaria alrededor del concepto de mediana. En P. Lestón (Ed.), Acta Latinoamericana de Matemática Educativa (Vol. 22, pp. 469-477). Ciudad de México: Comité Latinoamericano de Matemática Educativa.

MECD (2014). Real Decreto 126/2014, de 28 de febrero, por el que se establece el currículo básico de la Educación Primaria. Madrid: Ministerio de Educación, Cultura y Deporte.

MINEDU (2017). Programa curricular de Educación Primaria. Lima: Ministerio de Educación.

MINEDUC. (2012). Matemática educación básica. Bases curriculares. Santiago: Ministerio de Educación.

Mingorance, C. (2014). La estadística en las pruebas de diagnóstico andaluzas (Trabajo Fin de Grado). Universidad de Granada, España.

Neres, R. L. y Cantanhêde, R. B. S. (2018). Construindo conhecimento de média, mediana e moda: uma investigação docente. Cadernos de Pesquisa, 25(2), 187-206.

OCDE (2013). Marcos y pruebas de evaluación de PISA 2012: matemáticas, lectura y ciencias. Madrid: Ministerio de Educación, Cultura y Deporte.

Ocoró, L. V. y Ocoró, S. (2016). Análisis de las medidas de tendencia central en dos libros de textos escolares de grado séptimo: el caso de la media aritmética. En I. Álvarez y C. Sua (Ed.), Memorias del II Encuentro Colombiano de Educación Estocástica. Bogotá: Asociación Colombiana de Educación Estocástica.

Ortiz, J. J. (2002). La probabilidad en los libros de texto. Granada: Grupo de Investigación en Educación Estadística de la Universidad de Granada.

Ortiz, J. J., Font, V. y Mayén, S. (2009). Significados personales de la media aritmética de profesores en formación. En M.J. González, M.T. González y J. Murillo (Eds.), Investigación en Educación Matemática XIII (pp. 345-353). Santander: SEIEM.

Pérez-Serrano, G. (1994). Investigación cualitativa. Retos e interrogantes. I. Métodos. Madrid: La Muralla.

Rodríguez-Alveal, F., Díaz-Levicoy, D. y Maldonado-Fuentes, A. C. (2018). Evaluación del conocimiento y argumentación adquiridos por futuros profesores de secundaria de matemática sobre índices de resumen numérico. Investigación y Postgrado, 33(2), 97-114.

Rodríguez-Alveal, F., Maldonado-Fuentes, A. C. y Sandoval, P. (2012). Comprensión de las medidas de tendencia central: un estudio comparativo en estudiantes de pedagogía en matemática en dos instituciones formadoras chilenas. Avaliação: Revista da Avaliação da Educação Superior, 21(3), 929-952.

Salcedo, A., Molina-Portillo, E., Ramírez, T. y Contreras, J. M. (2018). Conflictos semióticos sobre estadística en libros de texto de matemáticas de primaria y bachillerato. Revista de Pedagogía, 39(104), 223-244.

SEP (2011a). Plan de estudios. Ciudad de México: Secretaría de Educación Pública.

SEP (2011b). Programa de estudios. Cuarto grado. Ciudad de México: Secretaría de Educación Pública. 
SEP (2011c). Programa de estudios. Quinto grado. Ciudad de México: Secretaría de Educación Pública.

SEP (2011d). Programa de estudios. Sexto grado. Ciudad de México: Secretaría de Educación Pública.

SEP (2014a). Desafíos Matemáticos. Libro para el alumno. Cuarto grado. Ciudad de México: Secretaría de Educación Pública.

SEP (2014b). Desafíos Matemáticos. Libro para el alumno. Quinto grado. Ciudad de México: Secretaría de Educación Pública.

SEP (2014c). Desafíos Matemáticos. Libro para el alumno. Sexto grado. Ciudad de México: Secretaría de Educación Pública.

SEP (2017). Aprendizaje clave para la Educación Integral. Plan y programa de estudio para la Educación Básica. Ciudad de México: Secretaría de Educación Pública.

Strauss, S. E. y Bichler, E. (1988). The development of children's concepts of the arithmetic average. Journal for Research in Mathematics Education, 19(1), 64-80. 